Revista Tecné, Episteme y Didaxis: TED. Año 2014, Número Extraordinario. ISSN Impreso: 0121-3814, ISSN web: 2323-0126

Memorias, Sexto Congreso Internacional sobre Formación de Profesores de Ciencias. 08 al 10 de octubre de 2014, Bogotá

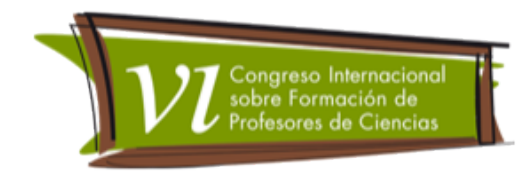

\title{
Unidades didácticas: Un camino para la transformación de la enseñanza de las ciencias desde un enfoque investigativo
}

Gallego Madrid, Diana Estella'; Quiceno Serna, Yesenia²; Pulgarín Vásquez, Darlin³.

Categoría 1. Reflexiones y experiencias desde la innovación en el aula.

\section{Resumen}

En la actualidad, la formación de maestros plantea retos importantes en relación a la comprensión de la realidad como una forma de generar propuestas de enseñanza contextualizadas que den respuesta a las necesidades del contexto educativo que se atiende.

Bajo el propósito de brindar una educación en ciencias de tipo integral, que más allá de alfabetizar en ciencia y tecnología pueda atender al desarrollo del pensamiento crítico de los estudiantes, la formación continua de maestros en la construcción de unidades didácticas con enfoque investigativo se convierte en el escenario propicio para articular procesos de investigación al desarrollo regular de las clases de ciencias, haciendo del acto de investigar, un ejercicio que posibilita tanto a estudiantes como a maestros la apropiación del conocimiento científico y la posibilidad de aplicarlo en su cotidianidad.

\section{Palabras claves}

Investigación escolar, formación continua, unidades didácticas con enfoque investigativo.

\section{Introducción}

La actividad científica puede entenderse como lo que los científicos hacen: mirar con otros ojos los fenómenos naturales mediados por ideas, conceptos, teorías, procedimientos, pero también mediante acciones como la indagación, la exploración, el cuestionamiento y la construcción de modelos explicativos sobre

${ }^{1}$ Licenciada en Ciencias Naturales y Educación Ambiental, UdeA, Candidata al título de Magíster en Educación, UdeA. Coordinadora académica, Parque Explora. gallediana@gmail.com, diana.gallego@parqueexplora.org

2 Licenciada en Ciencias Naturales y Educación Ambiental, UdeA, yesenia.serna@gmail.com

3 Licenciada en Ciencias Naturales y Educación Ambiental, UdeA, Magíster en Investigación en la Enseñanza y el Aprendizaje de las Ciencias Experimentales, Sociales y Matemáticas Universidad Internacional de Andalucía darlin20183@gmail.com 
Revista Tecné, Episteme y Didaxis: TED. Año 2014, Número Extraordinario. ISSN Impreso: 0121-3814, ISSN web: 2323-0126

Memorias, Sexto Congreso Internacional sobre Formación de Profesores de Ciencias. 08 al 10 de octubre de 2014, Bogotá

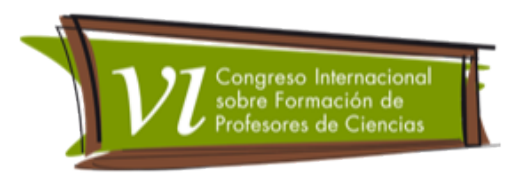

los fenómenos percibidos en el mundo real (Lineamientos Curriculares en Ciencias Naturales y Educación Ambiental, MEN 1998).

En este sentido, para enseñar ciencias naturales y generar aprendizajes en los estudiantes, se debe entender la ciencia como una disciplina dinámica, inacabada y en permanente cambio (Fourez, 1994), que posibilita el desarrollo de procesos de investigación de forma conjunta entre estudiantes y maestros.

Siendo consecuentes con las demandas de la educación en ciencias actual - El desarrollo del pensamiento crítico y la formación en ciudadanía- (Henao y Stipcich, 2008), es importante pensar en cómo los maestros pueden llegar a transformar su práctica docente al movilizar con sus estudiantes el desarrollo de habilidades científicas por medio de procesos de indagación e investigación en el aula.

Y es precisamente partiendo de las demandas de la educación en ciencias antes señaladas, que en la ciudad de Medellín y algunos municipios del Departamento de Antioquia, se ha venido desarrollando el programa de la Feria de la Ciencia, la Tecnología y la Innovación, el cual es liderado por la Alcaldía de Medellín, Empresas Públicas de Medellín - EPM y el Parque Explora desde el año 2008. De esta manera, en el marco de este programa -desde el año 2012- se vienen desarrollando espacios de formación continua de maestros en investigación escolar con dos módulos de formación, lo que ha permitido a los docentes participantes adquirir estrategias pedagógicas y didácticas que les permita dinamizar e innovar sus prácticas educativas.

A continuación se describe brevemente cada módulo de formación:

Módulo I: "Explorando-Preguntando": El docente se forma como facilitador y orientador del proceso de investigación que desarrollan sus estudiantes. Se espera que al final del módulo el docente realice una propuesta de investigación e incorporación al currículo desde el dispositivo de unidades didácticas.

Módulo II: "Encontrando-Comunicando": Dirigido a docentes que ya han iniciado procesos de investigación escolar (Docente Coinvestigador). Este docente tiene a su cargo el acompañamiento para el desarrollo de proyectos de investigación formulados por los equipos de estudiantes y la tarea de investigar como un miembro más de estos colectivos. 
Revista Tecné, Episteme y Didaxis: TED. Año 2014, Número Extraordinario. ISSN Impreso: 0121-3814, ISSN web: 2323-0126

Memorias, Sexto Congreso Internacional sobre Formación de Profesores de Ciencias. 08 al 10 de octubre de 2014, Bogotá

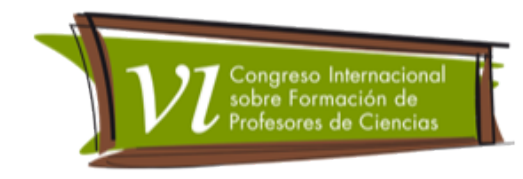

La experiencia presentada en este escrito, se enfoca en el proceso de formación vivido bajo el primer módulo de formación "Explorando-preguntando", en el cual los docentes diseñaron Unidades Didácticas con enfoque investigativo para su posterior intervención en el aula de clase.

\section{Desarrollo de la experiencia}

\section{¿Cómo se define y estructura una unidad didáctica convencional?}

Para promover una cultura basada en la generación, la apropiación y la divulgación del conocimiento, la investigación y el aprendizaje permanente de las ciencias, es necesario implementar un mecanismo de acción directa que permita a los maestros conectar los contenidos curriculares que se deben enseñar con los problemas de investigación que subyacen de cada contexto sociocultural.

Es así como el desarrollo de unidades didácticas, entendidas como "Unidades de programación y actuación docente, que da respuesta al qué enseñar (objetivos y contenidos), cómo enseñar (actividades, herramientas de enseñanza, organización del espacio y del tiempo, materiales y recursos didácticos) y cómo evaluar (criterios e instrumentos para la evaluación), se convierten en una herramienta que posibilita hacer de la investigación una acción posible dentro del currículo escolar (Mec, 1992, 87 o 91)".

Para el planteamiento de una unidad didáctica, se retoma la estructuración de la secuencia de actividades de enseñanza, el ciclo del aprendizaje de Jorba y Sanmartí (1996), Esquema 1. 
Revista Tecné, Episteme y Didaxis: TED. Año 2014, Número Extraordinario. ISSN Impreso: 0121-3814, ISSN web: 2323-0126

Memorias, Sexto Congreso Internacional sobre Formación de Profesores de Ciencias. 08 al 10 de octubre de 2014, Bogotá

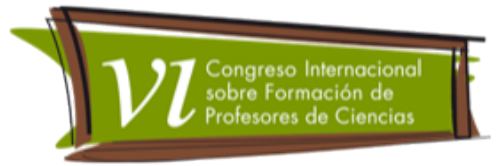

Esquema 1. El ciclo de aprendizaje de acuerdo con Sanmartí (1995) y con Jorba \& Sanmartí (1996)

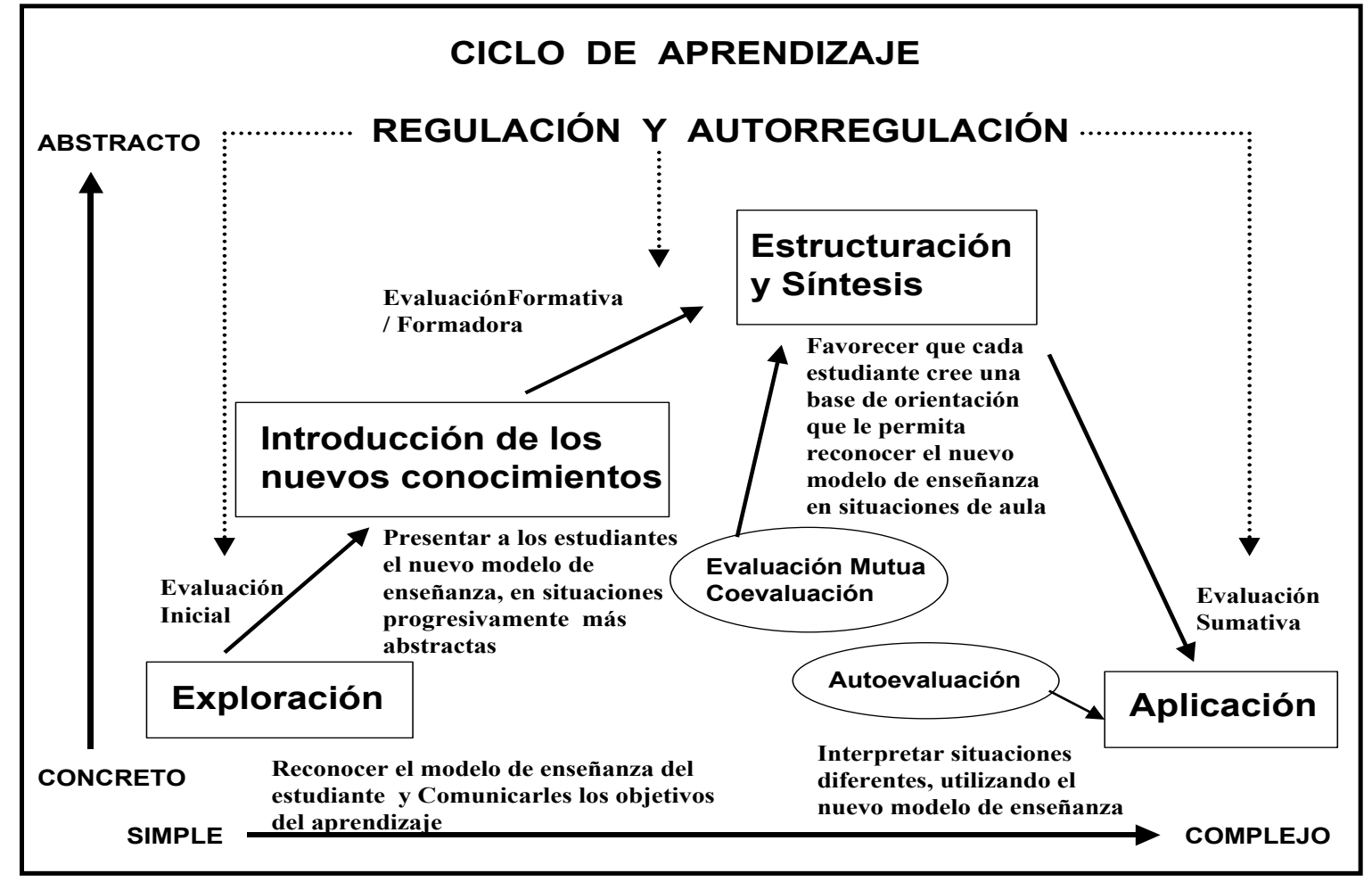

Desde el ciclo de aprendizaje, se configura cuatro fases, las cuales se presentan a continuación y a las que se integran los procesos de evaluación, regulación y autorregulación de los aprendizajes:

- Fase de exploración o de explicitación inicial: sitúa al estudiante en la temática objeto de estudio y busca captar su atención; a la vez que permite diagnosticar y activar conocimientos previos. En esta fase se desarrollan actividades que contribuyen a que los estudiantes formulen preguntas iniciales e hipótesis desde situaciones, vivencias e intereses cercanos.

- Fase de introducción de los nuevos conocimientos: orientada a observar, comparar o relacionar cada parte de lo que captó el estudiante inicialmente, de manera que estos se vean abocados a interactuar con el material de estudio, con sus pares y con el docente, buscando elaborar conceptos más significativos.

- Fase de estructuración y síntesis de los nuevos conocimientos: pretende ayudar al estudiante a construir el conocimiento como consecuencia de la interacción con el maestro, los compañeros y el ajuste personal. 
Revista Tecné, Episteme y Didaxis: TED. Año 2014, Número Extraordinario. ISSN Impreso: 0121-3814, ISSN web: 2323-0126

Memorias, Sexto Congreso Internacional sobre Formación de Profesores de Ciencias. 08 al 10 de octubre de 2014, Bogotá

- Fase de aplicación: permiten al estudiante aplicar los conocimientos adquiridos en otras situaciones similares.

A continuación, se presenta un esquema que relaciona los aspectos básicos que debe contener una unidad didáctica convencional integrada al ciclo del aprendizaje.

Esquema 2. Elementos de una unidad didáctica convencional
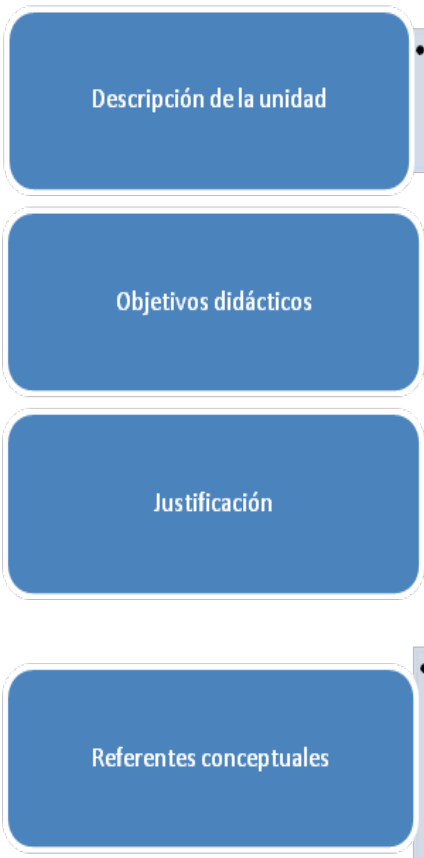

- Comprende un escrito, donde se relacionan los referentes que sustentan el desarrollo de los contenidos desde el saber disciplinar. Se debe entender muy bien, cuál es el fenómeno y explicarlo de forma resumida. También se entiende por referentes conceptuales, las perspectivas referidas por los autores al aprendizaje mismo (en este caso, al concepto de aprendizaje significativo, de unidades potencialmente significativas, metacognición, aprendizaje desde la perspectiva sociocultural, entre otras posibilidades). o nombre de la unidad; además, al número de sesiones de la unidad y el momento en que se va a poner en práctica,descripción de las competencias y estándares a desarrollar. y procedimental.

- En este apartado se trata de justificar por qué se ha escogido el tema y qué relación guarda con las necesidades o los intereses de los estudiantes que desarrollarán en la unidad.
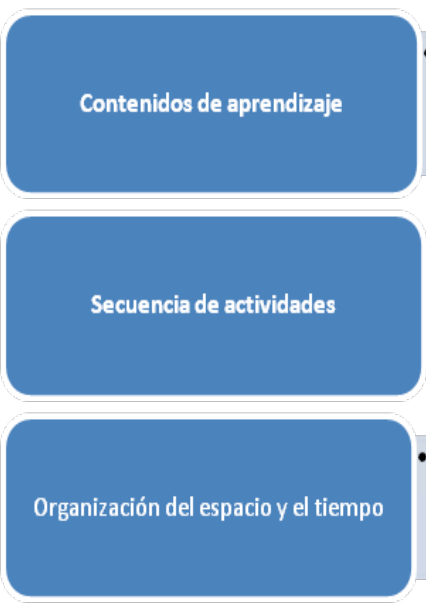

Evaluación
- En este apartado se podrá indicar la asignatura, el nivel/grado al que va dirigida, el tema en específico

- Los objetivos establecen qué es lo que en concreto, se pretende que adquiera el estudiante durante el desarrollo de la unidad didáctica. Es interesante explicitar los objetivos de tipo conceptual, actitudinal

- Se seleccionan ajustándolos a los objetivos planteados. Al hacer explícitos los contenidos de aprendizaje tanto conceptuales como procedimentales y actitudinales sobre los que se va a trabajar a lo largo del desarrollo de la unidad. Es importante que se determine también en el orden en que los estudiantes lo aprenderán.

- Pretenden facilitar la consecución de los objetivos y contenidos escogidos. En este apartado es muy importante establecer una secuencia de aprendizaje (ciclo del aprendizaje), en la que las actividades estén íntimamente relacionadas (exploración, introducción del nuevo conocimiento, estructuración y síntesis, aplicación).

- Se señalarán los aspectos específicos en tomo a la organización del espacio y del tiempo que requiera la unidad, y de manera específica, cada una de las actividades a desarrollar

- Las actividades que van a permitir saber sobre los aprendizajes de los alumnos, de la práctica docente del profesor y los instrumentos que se van a utilizar; deben situarse en el contexto general de la unidad, señalando cuáles van a ser los criterios e indicadores de valoración de dichos aspectos. 
Revista Tecné, Episteme y Didaxis: TED. Año 2014, Número Extraordinario. ISSN Impreso: 0121-3814, ISSN web: 2323-0126

Memorias, Sexto Congreso Internacional sobre Formación de Profesores de Ciencias. 08 al 10 de octubre de 2014, Bogotá

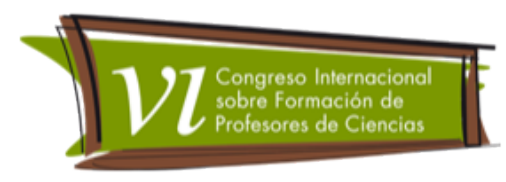

De esta manera la secuencia de actividades de las unidades didácticas abordadas en el proceso de formación de los maestros del Módulo I: ExplorandoPreguntando, se han organizado para su presentación utilizando como referente el ciclo de aprendizaje de Jorba y Sanmartí (1996), quienes proponen desde un modelo constructivista, identificar distintos tipos de actividades que tienen finalidades muy específicas.

\section{¿Qué elementos básicos están siendo considerados para el planteamiento de una propuesta investigativa desde el currículo?}

Desde la tendencia curricular investigativa planteada por Porlán (1993), se sugiere la construcción curricular desde los hechos culturales determinados por las características del contexto, que permita un equilibrio entre la disciplinariedad e interdisciplinariedad para asumir así procesos de investigación que compete a todos los miembros de la institución escolar. En esta misma tendencia, la ciencia es entendida como actividad cultural, en donde el conocimiento científico es una construcción humana que requiere consensos y acuerdos intersubjetivos, así como el reconocimiento de la investigación científica como plurimetodológica y que al igual que los métodos cuantitativos, se aceptan los métodos cualitativos en investigación.

De esta manera, el maestro ostenta autonomía y debe ser propositivo en su práctica de aula teniendo en cuenta que es el responsable de la enseñanza y de establecer una relación horizontal en donde se involucren los intereses de todos los miembros de la comunidad educativa, buscando también el equilibrio entre los referentes teóricos y la reflexión sobre su práctica, valiéndose de la investigación como proceso formativo en sus estudiantes y en la comunidad educativa. Es así como la enseñanza que imparte el maestro, gira en torno a proyectos a modo de pequeñas investigaciones o proyectos de investigación, como pretexto para facilitar el aprendizaje de sus estudiantes.

El proceso de aprendizaje busca el desarrollo integral y armónico del estudiante, donde éste se hace también responsable de su aprendizaje y su desarrollo cognitivo que incluye las dimensiones conceptual, metodológico y actitudinal, lo que conlleva a una evaluación como un proceso que incluye la valoración y continua cualificación de todos los elementos y procesos inherentes al currículo.

La experiencia que se retoma en esta propuesta para la trasformación de la enseñanza de las ciencias, se basa en la incorporación de la investigación escolar 
Revista Tecné, Episteme y Didaxis: TED. Año 2014, Número Extraordinario. ISSN Impreso: 0121-3814, ISSN web: 2323-0126

Memorias, Sexto Congreso Internacional sobre Formación de Profesores de Ciencias. 08 al 10 de octubre de 2014, Bogotá

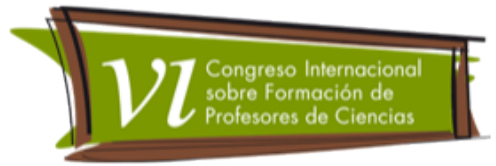

apartándose de posturas empiristas o positivistas que estarían reforzando visiones deformadas sobre la actividad científica, lo que se pretende resaltar es la reflexión y un pensamiento autónomo por parte de los estudiantes estableciendo puentes de lenguaje entre el conocimiento de sentido común con el conocimiento científico; lo cual, desde el ciclo didáctico y sus diferentes fases, aporta beneficios a los procesos de regulación y autorregulación del aprendizaje porque los educandos se involucran en su propio proceso de aprendizaje, su participación es activa y al ser reconocidos sus intereses se establece una conexión directa con el contexto y se empieza a relacionar la cotidianidad con lo trabajado en la clase de ciencias; por su parte, al maestro le posibilita establecer una relación entre elementos teóricos y prácticos facilitada por el trabajo de campo, la apertura hacia la indagación, las discusiones en común y la confrontación de los datos teóricos a la luz de lo obtenido durante la investigación, lo que a su vez, implica una transformación en la planeación curricular, puesto que el maestro también debe estar en constante reflexión y evaluación de los procesos de enseñanza y aprendizaje.

En el esquema 3, se evidencia cómo a partir de las diferentes fases del ciclo didáctico, se articulan los procesos de investigación de manera que, tanto la enseñanza de los contenidos (conceptuales, procedimentales y actitudinales propios de las ciencias naturales) y el aprendizaje, se presentan en la medida que se investiga y se indaga sobre los intereses particulares de los estudiantes.

Esquema 3. Ciclo de aprendizaje bajo un enfoque investigativo

CICLO DEL APRENDIZAJE PARA LA ELABORACIÓN DE UNIDADES DIDÁCTICAS CON ENFOQUE INVESTIGATIVO

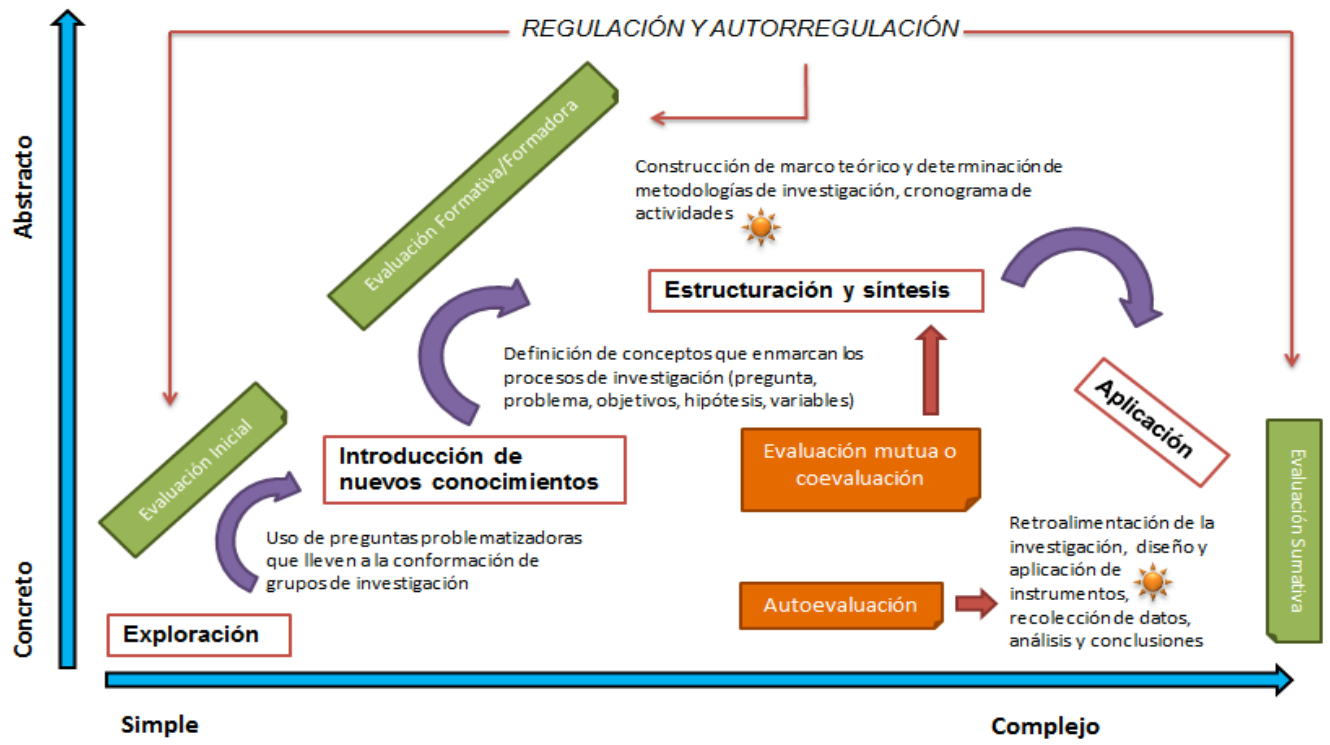


Revista Tecné, Episteme y Didaxis: TED. Año 2014, Número Extraordinario. ISSN Impreso: 0121-3814, ISSN web: 2323-0126

Memorias, Sexto Congreso Internacional sobre Formación de Profesores de Ciencias. 08 al 10 de octubre de 2014, Bogotá

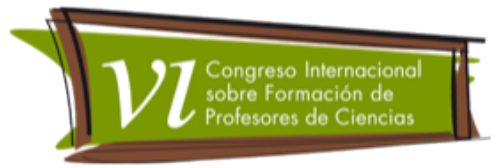

Se podría afirmar que durante el transcurso de la experiencia, identificar el valor de la investigación en el aula desde el ejercicio de planificación curricular, hace que los maestros centren la atención en el contexto escolar y los planes de estudio del área de ciencias naturales. Para este ejercicio es necesario que el enfoque investigativo se evidencie en la planificación y aplicación de la unidad didáctica y desde todos sus elementos constitutivos se pueda resaltar la indagación, la formulación de preguntas de investigación y la aplicación de diseños metodológicos investigativos. Así mismo, se busca que la planeación de estas unidades sea flexible para que se puedan abordar diferentes propuestas de investigación a partir de un eje temático central.

\section{La investigación escolar y las unidades didácticas}

La incorporación de procesos de investigación en las unidades didácticas diseñadas por los maestros participantes en la formación, permitió desencadenar procesos de investigación los sus estudiantes en el aula de clase, ya sea al inicio, durante o al final del desarrollo de la misma, los cuales se pueden presentar de la siguiente manera:

Esquema 4. Momentos de desarrollo de los procesos de investigación escolar articulados a las unidades didácticas

\begin{tabular}{|c|}
\hline $\begin{array}{l}\text { La investigación como eje del } \\
\text { diseño de la unidad didáctica: }\end{array}$ \\
\hline 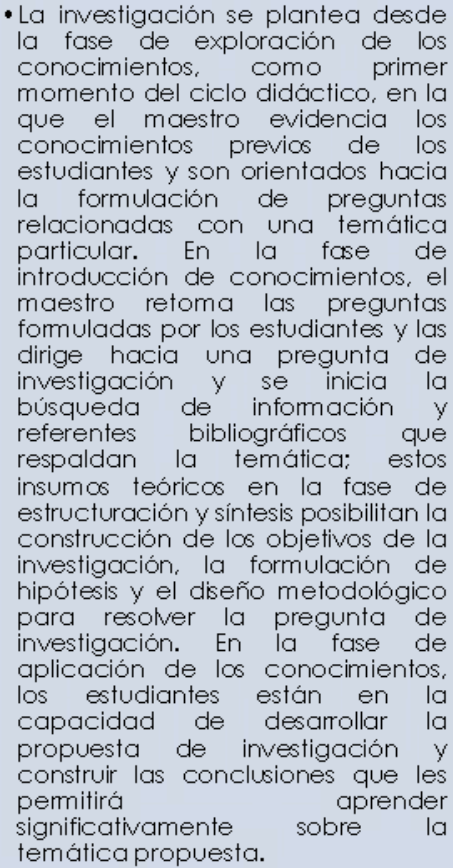 \\
\hline
\end{tabular}
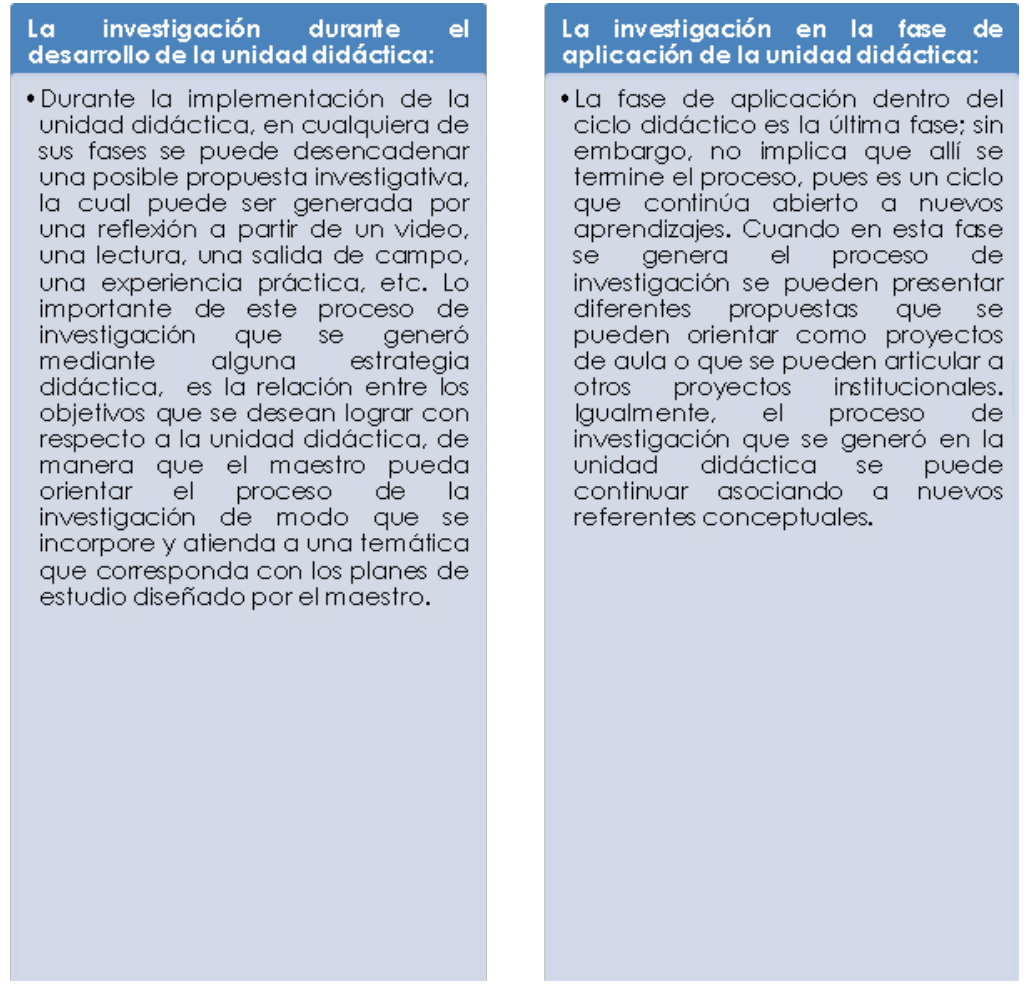
Revista Tecné, Episteme y Didaxis: TED. Año 2014, Número Extraordinario. ISSN Impreso: 0121-3814, ISSN web: 2323-0126

Memorias, Sexto Congreso Internacional sobre Formación de Profesores de Ciencias. 08 al 10 de octubre de 2014, Bogotá

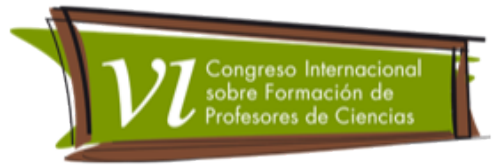

Lo anterior, representa las diferentes posibilidades que se pueden presentar en un proceso de enseñanza y aprendizaje cuando se aborda la investigación desde la programación del aula dentro del ciclo didáctico. Es de resaltar que la unidad didáctica es una vía para abordar la investigación, ya que ayuda a la comprensión de las relaciones complejas que se dan entre los fenómenos naturales y su influencia en el contexto sociocultural, económico, político y ambiental, además de permitir el desarrollo de competencias ciudadanas en la medida en que se trabaja en equipo, se socializan las propuestas de investigación y se presentan posibles soluciones para una problemática en particular.

\section{¿Cuáles son las habilidades investigativas que se puden desarrollar en los estudiantes y en los maestros?}

El proceso de investigación implica un trabajo colaborativo y el desarrollo de habilidades para la investigación que les posibilita tanto a maestros como estudiantes, indagar, discutir, reflexionar, comunicar, organizar información, establecer conexiones con otros campos de conocimientos y con otras personas o entidades que pueden aportar a los procesos de investigación.

Dentro del salón de clase, estas habilidades se adquieren en la medida que el docente asume un rol de facilitador, donde la información se despliega de forma multidireccional en la que los estudiantes también asumen diferentes roles, de acuerdo con sus habilidades (para indagar, organizar, comunicar, etc), igualmente, en el desarrollo de la investigación los maestros adquieren la habilidad para orientar los proyectos que surgen a partir de nuevos cuestionamientos, organizar, diseñar y ajustar la planeación de clase de acuerdo a los intereses de los estudiantes, gestionar recursos y apoyo académico con expertos que puedan aportar a la claridad conceptual de un tema específico.

En los estudiantes, las habilidades se van desarrollando desde un trabajo que involucre procedimientos, valores y actitudes sobre el tema a investigar, aspecto que dentro del ciclo didáctico, favorece en los estudiantes un constante análisis de la información y reflexiones sobre sus logros, dificultades y sobre aspectos que surgen dentro de la investigación, que asu vez, pueden desencadenar en otras indagaciones o reformulaciones sobre el trabajo desempeñado. Es posible que a partir de un proyecto de investigación surjan nuevas investigaciones y que estas se puedan focalizar bajo un mismo nucleo temático.

Cuando un docente, se inicia con sus estudiantes en la investigación, existe una fluidez en cuanto a la forma de hacerlo, de orientar el proceso, de modo que la 
Revista Tecné, Episteme y Didaxis: TED. Año 2014, Número Extraordinario. ISSN Impreso: 0121-3814, ISSN web: 2323-0126

Memorias, Sexto Congreso Internacional sobre Formación de Profesores de Ciencias. 08 al 10 de octubre de 2014, Bogotá

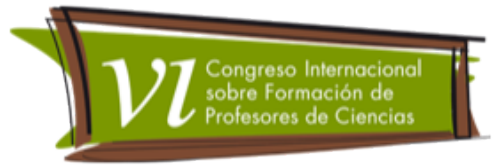

investigación está mediada por la habilidad de los estudiantes para indagar y preguntar, luego, el docente orienta y ofrece alternativas para el diseño metodológico de la investigación. Y los estudiantes, adicionalemente van desarrollando habilidades para los procesos orales y de lecto escritura.

\section{Experiencias significativas generadas en la formación de maestros en investigación escolar}

El primer reto de este proceso de formación continua de maestros, fue dilucidar las bondades de la planeación del trabajo de aula a través del diseño de unidades de enseñanza contextualizadas a las necesidades y realidades de la comunidad educativa. Subyacente a este arduo trabajo de naturaleza pedagógica, didáctica y disciplinar, sobreviene lo que implica para los maestros abandonar rutinas de enseñanza tradicionalistas que han sido validadas por la experiencia. Sin embargo, cuando los maestros desean generar cambios significativos en la forma de enseñar, se obtiene en estos un liderazgo que los posesiona en un ejercicio reflexivo y entre pares se apoyan las iniciativas de los maestros y se complementan los saberes, sobre experiencias significativas de aula, conocimeintos disciplinares, didácticos y pedagógicos.

La preparación y el diseño de unidades didácticas, ha puesto de manifiesto la resignificación del rol del docente, pues su desempeño en el aula adquiere reconocimiento por toda la comunidad educativa, los maestros buscan las rutas metodológicas para abordar la investigación y ésta se articula a los planes de estudio por medio de la indagación, la formulación de preguntas y el diseño de un enfoque metodológico.

Durante el ejercicio de construcción de unidades didácticas con enfoque investigativo, los maestros participantes gozaron de la libertad de diseñar propuestas de enseñanza en el marco de todos los núcleos temáticos a nivel curricular que presenta el área de ciencias naturales y educación ambiental. Como bien se señaló en los apartados anteriores, una de las características de éstas unidades es vislumbrar la conexión presente en si misma entre los planteamientos curriculares (qué contenidos enseñar) y la realidad próxima de los estudiantes. Para establecer esta relación, la investigación escolar actúa como puente entre el conocimiento científico y el conocimiento común del alumnado.

Las elaboraciones de los maestros dan cuenta que el currículo mismo brinda un abanico de posibilidades en el marco de la investigación escolar. Es posible valerse de sus mismos objetos de estudio y a través del tratamiento adecuado en 
Revista Tecné, Episteme y Didaxis: TED. Año 2014, Número Extraordinario. ISSN Impreso: 0121-3814, ISSN web: 2323-0126

Memorias, Sexto Congreso Internacional sobre Formación de Profesores de Ciencias. 08 al 10 de octubre de 2014, Bogotá

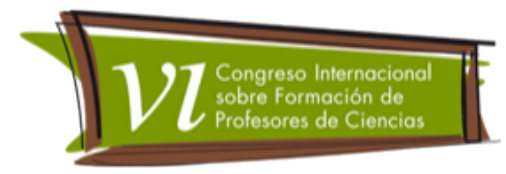

las aulas de clase, tornarlos objetos de investigación de gran impacto para las comunidades.

Al ser este ejercicio de construcción de unidades didácticas bajo este enfoque, el primero que de forma explícita atiende a la necesidad de incorporar la investigación escolar al desarrollo habitual de las clases de ciencias, algunos maestros participantes lograron incluir de forma articulada actividades que promueven la investigación escolar, mientras algunos incluyeron acciones complementarias a los contenidos evaluados y debatidos en las clases.

En relación a este aspecto, no se considera una dificultad la no articulación de estos dos componentes, pero se considera fundamental, a medida que estas propuestas toman lugar en las instituciones educativas, que ésta brecha existente entre enseñanza e investigación en ciencias comience a cerrarse, de tal forma que, la formación científica escolar asuma la investigación como un eje fundamental de la enseñanza.

\section{Conclusiones}

- En la formación con los maestros se reflexionó de manera constante sobre el rol del maestro investigador y el proceso de investigación desde el aula, así como las estrategias necesarias que el docente necesita para ampliar y enriquecer práctica. Así mismo, en la necesidad de profundizar en los aspectos metodológicos de la investigación y sus diferentes enfoques y cómo desarrollar el proceso de investigación con sus estudiantes que conlleve a la transformación de los esquemas tradicionales de enseñanza.

- Durante el diseño de las unidades didácticas, los maestros tuvieron espacios para la construcción individual, colectiva, la organización, selección y síntesis de la información suministrada sobre la temática. Igualmente, se reflexionó y valoró sobre el componente investigativo, evidenciándose una posición más amplia sobre la construcción del conocimiento científico. Además los maestros identificaron la viabilidad de desarrollar la unidad didáctica en el aula para desencadenar procesos investigativos, lo que también los condujo a la reflexión sobre la importancia de que el tema de investigación es más productivo cuando se toman decisiones grupales y cuando la elección de los estudiantes se privilegia ante los intereses del maestro. 
Revista Tecné, Episteme y Didaxis: TED. Año 2014, Número

Extraordinario. ISSN Impreso: 0121-3814, ISSN web: 2323-0126

Memorias, Sexto Congreso Internacional sobre Formación de Profesores de Ciencias. 08 al 10 de octubre de 2014, Bogotá

\section{Referencias bibliográficas}

Fourez, G (1994) La construcción del conocimiento científico, filosofía y ética de la ciencia. Narcea S.A de ediciones. Madrid. Pág. 206

Porlán, Rafael (1993) Constructivismo y escuela. Hacia un modelo de enseñanzaaprendizaje basado en la investigación, Sevilla, Díada editora. Pág. 194

Henao, B. y Stipcich, M. (2008). Educación en ciencias y argumentación: la perspectiva Toulminina como posible respuesta a las demandas y desafíos contemporáneos para la enseñanza de las ciencias experimentales. Revista Electrónica de Enseñanza de las Ciencias. 7 (1)47- 62 <Disponible en: http://reec.uvigo.es/volumenes/volumen7/ART3_Vol7_N1.pdf [Consulta: abril de 2014]

Jorba, J y Sanmartí, N (1996). Enseñar, aprender y evaluar: un proceso de evaluación continua. Propuesta didáctica para las áreas de ciencias de la naturaleza y las matemáticas. Barcelona. Ministerio de Educación y Cultura.

Ministerio de Educación Nacional (1998) Lineamientos Curriculares de Ciencias Naturales y Educación Ambiental. Men. Bogotá.

Ministerio de Educación y Cultura, España. Cajas rojas de educación primaria. Orientación y tutoría. Madrid, (Mimeo, Sin fecha). 